\section{La ética y la medicina social: la perspectiva de Michel Foucault}

\section{Ethics and social medicine: Michel Foucault's perspective}

\author{
Domingo Fernández Agis ${ }^{i}$ \\ i Profesor, Facultad de Humanidades, Sección de Filosofía/ \\ Universidad de La Laguna. \\ San Cristóbal de la Laguna - España \\ orcid.org/0000-0002-0702-1125 \\ dferagi@ull.edu.es
}

FERNÁNDEZ AGIS, Domingo. La ética y la medicina social: la perspectiva de Michel Foucault. História, Ciências, Saúde - Manguinhos, Rio de Janeiro, v.27, n.1, jan.-mar. 2020, p.171-180.

\section{Resumen}

La preocupación de Michel Foucault por la medicina, su historia y su impacto en la sociedad, es una constante en su obra. El objetivo de este trabajo es contrastar el contenido de las conferencias que Foucault impartió en Río de Janeiro, en octubre de 1974, con los materiales preparatorios de las mismas que forman parte de los fondos adquiridos por la Biblioteca Nacional de Francia. Una de las cuestiones clave en dichas conferencias es la relación entre la ética y la medicina social contemporánea. Esa cuestión, analizada desde el punto de vista de Foucault, constituye el trasfondo e interés último del presente trabajo.

Palabras clave: Michel Foucault (19261984); medicina social; ética; historia de la medicina.

\section{Abstract}

Michel Foucault's preoccupation with medicine, its history and its impact on society, is a constant in his work. The goal of this study is to contrast the content of the lectures Foucault gave in Rio de Janeiro, in October 1974, with the preparatory notes for them which are part of the archival holdings acquired by the National Library of France. One of the key questions in those lectures is the relationship between ethics and contemporary social medicine. This question, analyzed from Foucault's point of view, constitutes the background and ultimate interest of this article.

Keywords: Michel Foucault (1926-1984); social medicine; ethics; history of medicine. 
$\mathrm{L}$ a preocupación de Michel Foucault por la medicina, su historia y su impacto en la sociedad, es una constante en su obra. La vemos aparecer ya en sus primeros escritos, en los que la psiquiatría está tan presente. Baste recordar cómo, más allá de las reflexiones de naturaleza epistemológica que afectan al estatuto científico de disciplinas como la psiquiatría o la psicología, en Maladie mentale et personalité o, más tarde, en Histoire de la folie, Foucault se ocupa del impacto social de los conocimientos médicos o aplicados a la medicina. Años después, con una obra como Naissance de la clinique, realizará una aportación esencial a la historia de la medicina, pero no por ello dejará de lado la cuestión del impacto social de la medicina, que estará asimismo presente en obras tan aparentemente alejadas de este asunto como Surveiller et punir.

Precisamente, en la época en que está trazando y realizando el proyecto de esa extraordinaria obra, Foucault se muestra muy interesado en las cuestiones que vinculan ética y medicina, focalizando su interés sobre problemas sociopolíticos directamente vinculados a las mismas como intentaré mostrar en este trabajo.

Para ello, tomaré como base el establecimiento de un detenido contraste entre el contenido de las conferencias que Foucault impartió en Río de Janeiro, en octubre de 1974, y los materiales preparatorios de las mismas que forman parte de los fondos adquiridos por la Biblioteca Nacional de Francia. Una de las cuestiones clave en dichas conferencias es la relación entre la ética y la medicina social contemporánea. Esa cuestión, analizada desde el punto de vista de Foucault, constituye el trasfondo e interés último de estas páginas. Los asuntos abordados allí han tenido amplísimos desarrollos y siguen manteniendo en la actualidad un interés creciente, tanto por parte de los investigadores como por lo que respecta a los ciudadanos comunes que cada vez son más conscientes de la importancia que posee todo aquello que hoy podemos atinadamente considerar que ha quedado englobado en el ámbito de la biopolítica (Labisch, 1985, p.602-604).

\section{Foucault y la historia de la medicina social}

La caja n.8 del Fondo Michel Foucault, adquirido por la Biblioteca Nacional de Francia, contiene documentos relacionados con la historia de la medicina, la higiene, la policía de la salud y el papel de los profesionales de la medicina en la sociedad. Dentro de ella, la carpeta número 1 lleva como encabezamiento la anotación: "Histoire de la Médecine Social" y agrupa una serie de documentos preparatorios para las conferencias que Foucault pronunció en Río de Janeiro, en el mes de octubre de 1974. La primera de esas conferencias, "Crise de la médecine ou crise de la antimédecine?", fue publicada en 1976 (Foucault, 1994b). Rastreando el contenido de esas conferencias, pero sobre todo a través de los documentos preparatorios de las mismas, vemos cómo se va configurando una fuerte convicción, que permanecerá inamovible a partir de entonces, en el pensamiento de Foucault. En efecto, llegará a considerar incuestionable que, a partir de un determinado momento, la salud se convierte en una apuesta política o un juego político, en el sentido que hace uso, de forma reiterada, de ese término, vinculándolo siempre a los juegos y estrategias de poder. En palabras de Foucault, "la santé devienne enjeu politique" (Fonds..., 19.., caja 8, carpeta 1). Por otra parte, comprendemos la enorme amplitud que tenía para él todo el entramado de 
planteamientos e ideas, relacionados con la evolución de la medicina y su incidencia en la transformación de las tecnologías del poder (Hamraie, 2015, p.109).

En esa línea, ha de señalarse cómo Foucault se refiere en sus notas de trabajo a la utilización "eugenésica y racista" de ciertas "técnicas de la salud" (Fonds..., 19--, caja 8, carpeta 1). Este enfoque foucaultiano ha dado lugar a numerosos estudios, en los que la indagación acerca del origen y operatividad de la biopolítica conforman la base de un pensamiento social y político netamente crítico (Vázquez, 2009). Por su parte, Foucault considera que este aspecto tiene una gran importancia, pues pone de manifiesto un asunto que sigue siendo vital para nosotros; a saber, la proyección ético-política de la atención sanitaria (Fonds..., 19--, caja 8, carpeta 1). En efecto, el "derecho a la salud", se convierte, según nos dice Foucault, en las sociedades modernas y contemporáneas, en un problema político de primera magnitud. En ese sentido, toma en consideración en estos borradores, la relación entre el "devoir de santé" y el "droit de santé" (Fonds..., 19--, caja 8, carpeta 1), es decir, entre el deber del poder político de hacer todo lo necesario para asegurar la salud de los ciudadanos y el derecho de estos últimos a recibir toda la atención sanitaria que puedan necesitar. Foucault establece así una de las correlaciones más iluminadoras que puedan darse en relación a la política sanitaria, tan pertinente en el momento histórico en que aparece como en el mundo actual.

De una forma aún más explícita, escribe: "la salud se convierte en un objetivo político",1 situando por tanto la cuestión en ese terreno concreto de la acción política. Sin embargo, luego en sus apuntes, tacha la palabra "político" y la sustituye por la palabra "social". De esta forma, la frase queda configurada así: "la salud se convierte en un objetivo social" (Fonds..., 19--, caja 8, carpeta 1). Esto puede hacernos pensar en un retorno a la falta de concreción de la que tantas veces se acusa a la filosofía, pues decir que "la salud se convierte en un objetivo social" es difuminar el ámbito en el cual tenemos que centrar nuestra atención si queremos comprender los cambios en la política sanitaria que se han producido a partir del siglo XVIII. Esa evolución ha continuado hasta nuestros días (Castel, 1986, p.42-44). A este respecto, en la primera de sus conferencias de 1974, en Río de Janeiro, se referirá a que, a partir de los años 1940, "la salud se convierte en objeto de una verdadera lucha política", incidiendo sobre la "formulación de un nuevo derecho, una nueva moral, de una nueva economía, de una nueva política del cuerpo" (Foucault, 1994b, p.42).

\section{La política sanitaria: aspectos económicos}

No obstante, lo que Foucault quiere conseguir está bien lejos de un mero análisis histórico. En efecto, comprobamos a continuación que su objetivo es introducir la toma en consideración de los aspectos económicos en la política sanitaria. En ese sentido, realiza numerosas anotaciones relacionando la crisis económica y política, con la evolución de la medicina en el siglo XVIII. Así, aunque señala que esa crisis no ha vuelto a reproducirse con tal intensidad, no deja de tomar nota de la perdurabilidad de algunos de sus efectos. Esta cuestión será desarrollada en su ensayo "La politique de la santé au XVIIIe siècle" (Foucault, 1994a, p.14). No obstante, en este trabajo tiene aún más relevancia, a mi juicio, la cartografía que Foucault realiza de la "aparición de la salud y el bienestar físico de la 
población en general como uno de los objetivos esenciales del poder político" (Foucault, 1994a, p.6).

En sus notas de trabajo analiza asimismo el contenido y repercusiones del "Informe Beveridge", conocido bajo esa denominación al haber sido elaborado en 1942 por William Henry Beveridge, director de la London School of Economics. Este documento tiene una importancia trascendental, pues allí se recogen los planteamientos que marcarán la puesta en pie de la política sanitaria de los estados europeos a partir de entonces (Beveridge, 2012). Uno de los presupuestos básicos de los que se parte en el mencionado documento es que

el Estado puede ofrecer a la seguridad social sus servicios y su contribución financiera. Pero el Estado, al organizar la seguridad social, no debe matar en el individuo el incentivo, ni apartar de él la oportunidad, ni extinguirle su sentido de responsabilidad; una vez establecido un 'mínimun nacional', debe dejar libre el camino y estimular la acción espontánea de cada individuo con objeto de que mejore y supere para él y su familia ese mínimun (Beveridge, 2012, p.11).

Foucault analizará en detalle los análisis y propuestas del informe Beveridge y ese trabajo se verá reflejado en el contenido de su primera conferencia en Río de Janeiro, en 1974, donde el comentario del citado texto ofrece a Foucault (1994b, p.40-43) el marco discursivo apropiado para que él pueda exponer sus propias ideas de forma más elocuente y matizada. Le interesará especialmente, en este sentido, poner de relieve el doble juego de la política liberal, en lo que se refiere a la asistencia sanitaria de la población.

Se interesa asimismo por el "efecto patógeno de las técnicas médicas", refiriéndose en primer lugar a las técnicas que se utilizan con profusión en un momento dado y que, a continuación, dejan de emplearse. En ocasiones, tanto en su introducción como en su abandono, intervienen factores que tienen más que ver con la economía y la política sanitaria que con el valor terapéutico intrínseco de las aludidas técnicas. Señala además, a propósito de los resultados de su aplicación con vistas al avance del conocimiento médico, la imposibilidad de utilizar en el futuro los datos obtenidos a partir de ellas. Con lo que el impacto negativo del citado proceso se multiplica.

\section{Medicina y biopolítica}

Por otra parte, en el trabajo que acabamos de citar, Foucault analizará la conversión de la medicina en una "técnica general de la salud" (Foucault, 1994a, p.23). Ello la convertirá en un recurso de inapreciable valor para poner en marcha o hacer aún más efectivas ciertas estrategias de poder. No obstante, como cualquier otra técnica, también la aplicación de los cuidados médicos a la población está sujeta a disfuncionalidades y desequilibrios. Una relevante ilustración literaria de esto la podemos encontrar en la experiencia personal de convivir con la enfermedad y su tratamiento, tal y como ésta es narrada por el escritor y amigo de Foucault, Hervé Guibert, en sus obras tituladas, A l'ami qui ne m'a pas sauvé la vie y Le protocole compassionnel. ${ }^{2}$ En ellas encontramos la descripción pormenorizada de las crecientes sospechas del paciente, a propósito de la eficacia y sentido de algunas de las 
técnicas que se le aplicaron para tratar de frenar la progresión de la enfermedad del Sida, que, al igual que le sucedió a Foucault, Guibert (1990, p.167) padecía.

En la conferencia a la que me estoy refiriendo, el filósofo hace una detallada alusión a los efectos negativos de determinados tratamientos médicos, poniendo de relieve la ineficacia y aún la maleficencia de algunos medicamentos que, sin embargo, siguen empleándose de forma masiva (Foucault, 1994b, p.5-7). En esa misma línea, es preciso destacar también su inquietud a propósito de las contrapartidas y peligros de los avances de ese campo puntero en la biomedicina que es la ingeniería genética (p.48). Es consciente, en la época en que se están produciendo los avances decisivos en las técnicas de manipulación genética, de los problemas éticos que se van a suscitar al dejar atrás definitivamente la frontera que se está atravesando. Además de ello, es capaz de poner en perspectiva tales avances, situándolos en el contexto de la transformación del cuerpo en un objeto manipulable y transformable por la acción humana.

Foucault habla, en este sentido, de la transformación del cuerpo en objeto, en puro elemento material, en la medicina del siglo XVIII. En relación a ello, hay en estos apuntes una página tachada por la mano de Foucault que tiene gran interés. En ella había anotado de forma breve y algo abrupta una serie de ideas que irá desarrollando a lo largo de cursos y conferencias posteriores. Habla así de la transformación de la guerra, deviniendo lo "social o racial" el centro de la misma, a lo largo del siglo XIX. Nos dice que, con ella, el riesgo de retroceso a la barbarie y la oposición radical entre vida y muerte, "vuelve a la superficie"3 (Fonds..., 19--, caja 8 , carpeta 1). Esta anotación tiene una apreciable relevancia, ya que el filósofo está apuntando hacia aspectos que, en efecto, son cruciales en los conflictos bélicos de esa época y, sobre todo, en las actuales confrontaciones. Dichos aspectos alejan la solución de los mismos del escenario ideal, definido por el imperio del derecho internacional.

Se plantea asimismo la necesidad de explorar dónde radica el punto central de las nuevas batallas, aquello que podría permitir la comprensión de sus causas últimas. En ese sentido, remite a la problematización de las relaciones entre el saber y el poder, con la consiguiente consideración de "malos saberes", que recae sobre aquellas formas de conocimiento que no son sumisas al poder o útiles al mismo. Según nos dice, a partir del siglo XIX hemos descubierto, con Darwin y Virchow, la "potencia indefinida e inagotable" de la vida, pero también la amenaza permanente a la que la vida se halla sometida (Fonds..., 19--, caja 8, carpeta 1). Se produce, de igual manera, una cierta "matematización de la vida y la muerte" (Frioux, Fournier, Chauveau, 2011, p.74), que Foucault tendrá muy presente cuando se ocupe de estudiar aspectos como los relativos a la alimentación y la salud de la población o, en general, a "la gubernamentalidad", en su curso, Sécurité, territoire, population (Foucault, 2004, p.42).

\section{Derecho a la salud y medicalización de la sociedad}

También presta atención, en otras páginas que encontramos entre sus notas de trabajo, a la crisis actual de la medicina, en el contexto de sus referencias al derecho a la salud y sus consecuencias. Desde esa perspectiva, se refiere a los problemas que tienen las sociedades 
a partir de cierta etapa de su desarrollo y que afectan a la política sanitaria, como los problemas derivados de la sobrepoblación. En este sentido, es importante traer a colación el contenido de otro de los cursos que impartió en el Collège de France, además del que mencionábamos más arriba, cuya trascendencia ha sido muy apreciable en el ámbito de los estudios foucaultianos, desarrollado bajo el título de Naissance de la biopolitique (Sorrentino, 2012, p.40-41). Como es sabido, en ambos cursos aborda de una manera detenida la correlación entre política de la salud y política de la población. En particular en éste último, al analizar los cambios que conlleva la introducción de la economía política liberal (Foucault, 2004, p.34-35).

En todo caso, en la primera de sus intervenciones en Río de Janeiro en 1974, conferencias que es necesario tener siempre presentes en este trabajo como elementos básicos de correlación, Foucault (1994b, p.48) habla del "fenómeno de la medicalización indefinida de la sociedad". Este fenómeno es característico de la medicina del siglo XX y sigue siendo una característica esencial de la relación de los ciudadanos con los cuidados médicos en el siglo XXI, en los países occidentales.

Aún así, es de apreciable relevancia que en sus anotaciones se aproxime a la influencia ejercida, a partir del siglo XIX, por el evolucionismo en su versión darwiniana. Esto le permite abordar la cuestión de la toma en consideración de la salud de la especie y de la atención a todo aquello que pueda ayudar a la superación de las taras de origen biológico. Estos planteamientos nos abren, en consecuencia, a las políticas eugenésicas, cuya presencia es tan notoria como lamentable en la primera mitad del siglo XX (Esposito, 2006, p.17). Con respecto a ellos, se nos plantea la necesidad de distinguir entre lo que el discurso del poder parece decir y lo que "dice", en realidad. En este sentido, la labor de estudio histórico confluye con el trabajo de esclarecimiento epistemológico y de análisis político (Lorenzini, 2014, p.13).

En todo caso, vemos cómo el planteamiento de Foucault vira hacia la toma en consideración de los aspectos sociales de la medicina. Para él, queda claro que el giro político que se da con la atención a la salud de las poblaciones conduce a considerar la enfermedad, no tanto como algo que afecta a individuos concretos, es decir, que es un problema particular de los enfermos que la padecen, sino como una realidad que ha de mantenerse bajo control ya que puede suponer una amenaza para la sociedad (Frioux, Fournier, Chauveau, 2011, p.38-39).

En ese contexto, alude a la preocupación por la higiene pública, refiriéndose a la toma en consideración de problemas como el de la polución del aire y el agua, los ritmos de trabajo o los hábitos de comportamiento. Todos estos asuntos tienen, sin duda, una dimensión económica, pero su proyección política es más importante aún. En atención a la importancia de estas cuestiones, Foucault explora el contenido de diversos documentos concernientes a la política de la salud durante los siglos XVIII y XIX. Es interesante, a este respecto, recorrer su exploración del contenido de periódicos y revistas vinculados a la causa obrera que, durante el siglo XIX, se hacen eco de la necesidad de mejorar las condiciones de trabajo, alimentación e higiene de la población trabajadora, así como la habitabilidad de los lugares en que ésta reside (Fonds..., 19--, carpeta 1). 


\section{El cuerpo como objeto político y económico}

En la carpeta número 2, encontramos una serie de anotaciones referidas al cuerpo y su toma en consideración como objeto político y económico. Foucault habla de la influencia que la economía capitalista ha ejercido sobre el desarrollo de la medicina. A su juicio, la mercantilización de la sociedad ha transformado el modo y el sentido mismo del ejercicio de la medicina. Por otra parte, aborda una serie de reflexiones que pretenden esclarecer las posibilidades que la medicina social ha ofrecido a la medicina clínica (Fonds..., 19--, caja 8, carpeta 2). Estas transformaciones se ponen de relieve con objeto de abocar a una conclusión singular, pues para Foucault el cuerpo adquiere un valor sociopolítico, más allá de su realidad biológica. Enlazando con ello, en una frase algo enigmática, se refiere a que "nuestro humanismo es reticente", ${ }^{4}$ señalando así la persistencia de la disociación entre la interpretación científica del cuerpo, con sus inevitables derivas políticas y económicas, y la concepción humanista que se resiste a desaparecer, pese a no poder dar cuenta de los juegos de poder en los que la corporeidad se ve envuelta en el mundo de hoy (Fernández Agis, 2007, p.87 y s.). Foucault ve, sobre todo, en la propia evolución del conocimiento médico la razón más clara de esa reticencia humanista (Fonds..., 19--, caja 8, carpeta 2).

Nos encontramos también con una serie de apuntes sobre "La salubrité des villes", donde da cuenta de los cambios urbanísticos que, con la finalidad de mejorar la salubridad de las ciudades, se emprenden a partir del siglo XVIII en distintas ciudades de Europa. En concreto, nos muestra cómo en París se fragua y empieza a utilizar la imagen de la ciudad como un cuerpo, cuyo bienestar y salud hay que preservar. En cierta manera puede hablarse, en este sentido, de la conversión de esta ciudad en algo así como un "laboratorio", para la experimentación del urbanismo higienista (Frioux, Fournier, Chauveau, 2011, p.84-85). En todo caso, tal idea de fondo se concreta en la apelación a un "corps urbain cohérent" (Fonds..., 19--, caja 8, carpeta 2). Esta alusión a la coherencia del "cuerpo urbano", no debe ser pasada por alto. En efecto, Michel Foucault se detiene a comentar la influencia del urbanismo y realiza un contraste entre la ciudad moderna y la ciudad medieval. Reflexiona acerca de los miedos que en los siglos precedentes suscitaban las ciudades, consideradas como lugares en los que era más fácil y frecuente la transmisión de enfermedades. Más tarde, el miedo que se tenía a la ciudad será sustituido por el miedo a todo lo que viene de fuera de ella. Pero, durante mucho tiempo será frecuente, en palabras de Foucault, "un miedo a la ciudad, reemplazado por miedo al campesinado"5 (Fonds..., 19--, caja 8, carpeta 2). Nos encontramos, por tanto, con una toma en consideración de los riesgos de la vida urbana debidos a los problemas derivados de la concentración de individuos en un espacio reducido. Queda establecida de forma forzosa una vinculación entre urbanismo y medicina. Foucault ve en ello una de las claves para comprender la aparición de la medicina social. Siguiendo, como él mismo reconoce, la estela de los estudios foucaultianos, Richard Sennett (1997, p.20-21) abundó en este mismo tema en su excelente obra titulada Carne y piedra: el cuerpo y la ciudad en la civilización occidental. En ella podemos encontrar expuesta de una forma muy elocuente la correlación entre la evolución de la vida urbana y algunas de las patologías que afectan al ser humano en la actualidad (p.23). 


\section{Higiene pública y control social}

Un aspecto esencial es el de la higiene, estableciéndose distintas formas de correlación entre la limpieza del individuo, la pulcritud del ambiente, la idoneidad de las condiciones de vida, la pureza del agua consumida, la alimentación y la salud. En este contexto, Foucault habla de la importancia del "modelo de la peste" en la constitución de las políticas que tienen como centro la higiene pública. Por tanto, apela a un modelo centrado en el aislamiento del foco de contagio de la enfermedad y al férreo control "policial" de la población. Se refiere también a los cementerios, que serán objeto de una atención sanitaria sin precedentes a finales del siglo XVIII (Fonds..., 19--, caja 8, carpeta 2). En efecto, en esa época fueron vistos como focos potenciales de difusión de enfermedades, por lo que se establecieron una serie de disposiciones normativas destinadas a regular su ubicación y su uso. De igual manera, nos muestra en detalle cómo, en esta época, empieza a considerarse un problema digno de atención el de la "salud de los pobres" (Fonds..., 19--, caja 8, carpeta 2). Por ello, Foucault estudia numerosos documentos relativos a esta cuestión, dejando también un importante rastro documental de dichos estudios. Al hilo de esos estudios, muestra una vez más su discrepancia con el marxismo que, a su juicio, proporciona una útil metodología de análisis, pero no un enfoque adecuado para resolver los problemas sobre los que centra su atención (Fonds..., 19--, caja 8, carpeta 2).

Recopila asimismo gran cantidad de documentación relacionada con la medicina actual. Entre ella, tienen especial interés e indudable actualidad sus anotaciones a propósito de la industria farmacéutica, que ponen de manifiesto la dependencia de las multinacionales del sector. Al mismo tiempo, permiten mostrar las profundas implicaciones económicas que tiene todo cuanto guarda relación con la elaboración, distribución y venta de los medicamentos en la sociedad actual.

Encontramos, asimismo, un interesante dossier que, bajo el epígrafe de "Le médecin" nos ofrece esclarecedoras reflexiones de Foucault sobre la evolución de la figura del médico en las sociedades occidentales, sobre todo desde los inicios del siglo XIX. En esas anotaciones habla, en primer término, del uso de los medicamentos, en el contexto del proceso de normalización de la medicina que de forma tan decisiva ha marcado el desarrollo del saber y la praxis médica contemporánea. Este proceso supone una relación específica entre la medicina y la técnica. Al mismo tiempo, la relación del médico con los medicamentos es muy diferente de la que se daba en la medicina anterior. Foucault habla de "hacer valer el carácter semiológico del medicamento"6 (Fonds..., 19--, caja 8, carpeta 2), introduciendo así una referencia a la definición y caracterización de la enfermedad en el interior de un discurso médico que va construyendo una semiología novedosa y específica. Por otra parte, se refiere a un aspecto que puede parecer chocante, ya que habla del medicamento haciendo alusión a "su carácter de vehículo de poder"7 (Fonds..., 19--, caja 8, carpeta 2). Piensa, en efecto, en la utilización política de la medicalización. No en el sentido de posibilitar a través de ella la transformación de la población en un colectivo sumiso, sometido a los imperativos de la adicción, sino aludiendo a formas de sumisión más sutiles, aunque quizá más eficaces para el mantenimiento de las relaciones de poder. Decididamente, a través de la medicalización de la población podemos comprobar que, en efecto, la salud se ha convertido en parte de un gran juego político. 


\section{Consideraciones finales}

Con la finalidad de recoger algunas ideas clave, que a su vez sintetizan en buena medida el recorrido que hemos seguido hasta aquí, me referiré en estas líneas finales a la segunda conferencia impartida por Foucault en Río de Janeiro en 1974, que llevaba por título "Naissance de la médecine sociale", aludiendo así, de forma más directa, al asunto que nos ha ocupado en estas páginas. En ella el pensador confirma que

sostiene la hipótesis de que, con el capitalismo, no se ha pasado de una medicina colectiva a una medicina privada, sino que se produce precisamente todo lo contrario; el capitalismo que se desarrolla a finales del siglo XVIII y principios del siglo XIX, ha socializado de entrada un primer objeto, el cuerpo, en función de la fuerza productiva, de la fuerza de trabajo. El control de la sociedad sobre los individuos no se efectúa solamente por la conciencia o la ideología, sino también en el cuerpo y con el cuerpo. Para la sociedad capitalista, es lo bio-político lo que importa, ante todo, lo biológico, lo somático, lo corporal (Foucault, 1994c, p.209-210).

Por eso Foucault (1994c, p.210) concluye que "el cuerpo es una realidad biopolítica; la medicina, una estrategia biopolítica".

Emerge de esta forma un enfoque nuevo de muchas cuestiones que, durante siglos, han sido objeto de atención en el ámbito económico y en el propio del poder político, allí donde ambos pueden separarse. El punto nodal de este nuevo enfoque radica en la centralidad que se concede al cuerpo, ya que las estrategias del poder biopolítico se diseñarán partiendo del conocimiento científico del cuerpo. Este conocimiento, en consecuencia, es la base de toda proyección del poder biopolítico sobre los individuos y la sociedad.

\section{NOTAS}

1 "La santé devienne un objectif politique."

${ }^{2}$ Ambas publicadas en español por la editorial Tusquets, bajo los siguientes títulos: Al amigo que no me salvó la vida y El protocolo compasivo.

3 "Reviens à la surface."

4 "Notre humanisme est réticent."

5 "Une peur de la ville, que se substitue à la peur de la paysannerie."

6 "Faire valoir le caractère semiologique du médicament."

7 "Son caractère de vehicule de pouvoir."

\section{REFERENCIAS}

BEVERIDGE, William.

La seguridad social en Inglaterra: Plan Beveridge. Ciudad de México: CIESS. 2012.

CASTEL, Robert.

Les aventures de la pratique. Le Débat, n.41, p.41-51. 1986.

ESPOSITO, Roberto.

Bíos: biopolítica y filosofía. Buenos Aires:

Amorrortu. 2006.
FERNÁNDEZ AGIS, Domingo.

Michel Foucault, ética y política de la corporeidad. Santa Cruz de Tenerife: Idea. 2007.

FONDS...

Fonds Michel Foucault. Département de Manuscrits, NAF 28730, caja 8 (Bibliotèque National de France, Paris). [19--]. 
FOUCAULT, Michel.

Naissance de la biopolitique. Paris: Gallimard; Seuil. 2004.

FOUCAULT, Michel.

La politique de la santé au XVIIIe siècle. In: Foucault, Michel. Dits et écrits. t.3. Paris:

Gallimard. p.725-741. 1994a.

FOUCAULT, Michel.

Crise de la médecine ou crise de la antimédecine? In: Foucault, Michel. Dits et écrits. t.3. Paris: Gallimard. p.40-57. 1994b.

FOUCAULT, Michel.

Naissance de la médecine sociale. In: Foucault, Michel. Dits et écrits. t.3. Paris: Gallimard. p.207227. 1994c.

FRIOUX, Stèphane; FOURNIER, Patrick; CHAUVEAU, Sophie.

Hygiène et santé en Europe: de la fin du XVIIIe siècle aux lendemains de la Première Guerre Mondiale. Paris: Sedes. 2011.

GUIBERT, Hervé.

A l'ami qui ne m'a pas sauvé la vie. Paris: Gallimard. 1990.
HAMRAIE, Aimie.

Historical epistemology as disability studies methodology: from the models framework to Foucault's archaeology of cure. Foucault Studies, n.19, p.108-134. 2015.

LABISCH, Alfons.

Doctors, workers and the scientific cosmology of the industrial world: the social construction of 'health' and the 'homo hygienicus'". Journal of Contemporary History, v.20, n.4, p.599-615. 1985.

LORENZINI, Daniele.

La politique des conduites: pour une histoire $\mathrm{du}$ rapport entre subjectivation éthique et subjectivité politique. Thèse (Doctorat) Université Paris-Est Créteil. 2014.

SENNETT, Richard.

Carne y piedra: el cuerpo y la ciudad en la civilización occidental. Madrid: Alianza. 1997.

SORRENTINO, Vincenzo.

Bipolitica, libertad e cura. Roma: Aracne. 2012.

VÁZQUEZ, Francisco.

La invención del racismo: nacimiento de la biopolítica en España. Madrid: Akal. 2009. 